\title{
Retraction Note to: Chrysophanol exhibits anti-cancer activities in lung cancer cell through regulating ROS/HIF-1a/VEGF signaling pathway
}

\author{
Jie Zhang ${ }^{1} \cdot$ Qian Wang $^{1,2} \cdot$ Qiang Wang ${ }^{1} \cdot$ Peng Guo ${ }^{1} \cdot$ Yong Wang ${ }^{1} \cdot$ Yuqing Xing ${ }^{1} \cdot$ Mengmeng Zhang $^{1} \cdot$ \\ Fujun Liu ${ }^{1} \cdot$ Qingyun Zeng ${ }^{2}$
}

Published online: 26 November 2020

(C) Springer-Verlag GmbH Germany, part of Springer Nature 2020

\section{Retraction Note to: Naunyn-Schmiedeberg's Archives of Pharmacology (2020) 393:469-480 https://doi.org/10.1007/s00210-019-01746-8}

The Editor in Chief retracted this article (Zhang et al. 2020) because of significant concerns regarding a number of Figures presented in this work. After publication, overlap was detected with figures in unrelated articles, some published previously and others within a close time frame.

Figure 2A appears to contain the same images that have been published elsewhere with different labels: Figure 6K (Yin et al. 2020) and Figure 3E ((Liu 2018), retracted).

Figure 2E images labelled as A549 cells appears to be similar to that published elsewhere with different labels: Figure $4 \mathrm{~K}$ (Yin et al. 2020) and Figure 2E (Wang et al. 2018).

Figure $3 \mathrm{G}$ - overlap in images used in this and another unrelated article by other authors (Wang et al. 2019)

Figure 6A appears to contain images that have been published elsewhere with different labels: Figure 8B in ((Liu 2018), retracted), Figure 6A (Sun et al. 2020), Figure 7A in (Niu et al. 2019), and Figure 6A ((Gao 2017), retracted).

The online version of the original article can be found at https://doi.org/ 10.1007/s00210-019-01746-8

Qingyun Zeng

qingyunzengjnsd@163.com

Qiang Wang

qingyunzengjnsd@163.com

1 Shandong Provincial Hospital affiliated to Shandong University, Jinan 250021, Shandong, China

2 Hospital Affiliated to Shandong University of Traditional Chinese Medicine, Jinan 250011, Shandong, China
Figure 6E appears to contain images that have been published elsewhere with different labels: Figure 6D (Sun et al. 2020) and Figure 6C (Han et al. 2019).

Figure $6 \mathrm{~K}$ appears to contain images that have been published elsewhere with different labels (Sun et al. 2020)

The authors were unable to provide raw data and stated that the figures were produced by a commercial lab hired to perform some of the experiments for this study. Furthermore, the authors stated that some of the data presented were not generated as part of this study. The Editor-in-Chief therefore no longer has confidence in the integrity of the data in this article. All authors agree to this retraction.

\section{References}

Retracted article: Gao MQ et al. (2017) Hispidulin suppresses tumor growth and metastasis in renal cell carcinoma by modulating ceramide-sphingosine 1-phosphate rheostat. Am J Cancer Res 7(7): 1501-1514

Han M, Gao H, Xie J et al (2019) Hispidulin induces ER stress-mediated apoptosis in human hepatocellular carcinoma cells in vitro and in vivo by activating AMPK signaling pathway. Acta Pharmacol Sin 40:666-676. https://doi.org/10.1038/s41401-018-0159-7

Retracted article: Liu K. et al. (2018) Hispidulin suppresses cell growth and metastasis by targeting PIM 1 through JAK 2/STAT 3 signaling in colorectal cancer. Cancer Sci. https://doi.org/10.1111/cas.13575

Niu Y et al (2019) Physcion 8-O- $\beta$-glucopyranoside induced ferroptosis via regulating miR-103a-3p/GLS2 axis in gastric cancer. Life Sciences V237:116893. https://doi.org/10.1016/j.lfs.2019.116893

Sun Y, Lv B, Zhang X (2020) Knock-down of LncRNA-XIST induced glioma cell death and inhibited tumorigenesis by regulating miR137/SLC1A5 axis-mediated ROS production. NaunynSchmiedeberg's Arch Pharmacol. https://doi.org/10.1007/s00210020-01831-3

Wang N et al (2018) Euxanthone suppresses tumor growth and metastasis in colorectal cancer via targeting CIP2A/PP2A pathway. Life Sci 209:498-506. https://doi.org/10.1016/j.lfs.2018.08.052 
Wang L, Wang J, Zhao H et al (2019) Soyasapogenol B exhibits antigrowth and anti-metastatic activities in clear cell renal cell carcinoma. Naunyn-Schmiedeberg's Arch Pharmacol 392:551-563. https:// doi.org/10.1007/s00210-018-01607-w

Y in J, Yin Q, Liang B et al (2020) Chrysophanol suppresses growth and metastasis of $\mathrm{T}$ cell acute lymphoblastic leukemia via miR-9/PD-L1 axis. Naunyn-Schmiedeberg's Arch Pharmacol 393:273-286. https://doi.org/10.1007/s00210-019-01778-0
Zhang J, Wang Q, Wang Q et al (2020) Chrysophanol exhibits anticancer activities in lung cancer cell through regulating ROS/HIF1a/VEGF signaling pathway. Naunyn-Schmiedeberg's Arch Pharmacol 393:469-480. https://doi.org/10.1007/s00210-01901746-8

Publisher's note Springer Nature remains neutral with regard to jurisdictional claims in published maps and institutional affiliations. 